\title{
Design and Architecture of GIS Servers for Web Based Information Systems - The ArcGIS Server System
}

\author{
Sudhakar Menon \\ ESRI \\ Redlands, CA, USA \\ smenon@esri.com
}

This talk provides an overview of the design and architecture of GIS Servers for web based information systems, using ESRI's ArcGIS Server system as a concrete example. A GIS Server allows customers to compile, manage and disseminate geographic information. Information is compiled into and stored within object relational spatial databases using a geodatabase information model that supports the key types needed by applications including features, relationships, networks, imagery, terrains, maps and layers. Information is managed using both short and long transaction models that include support for versioning, archiving and replication. The GIS Server allows administrators to selectively publish this information to clients using stateless web services based on REST and SOAP as well as via OGC interfaces. These geospatial web services support visualization, analysis, data access and replication. Key GIS services include Mapping, Query, Location, Network Analysis, Editing, Geoprocessing and Imaging. These services run on clusters, can access data from local caches, and can be scaled out by growing the cluster. These web services allow information to be exchanged using optimized representations based on JSON and XML as well as client specific optimized protocols. The GIS Server supports a role based authorization model that allows access to services to be controlled, leverages standard web based authentication mechanisms, and allows information to be securely exchanged by leveraging standard transport level security. The GIS Server supports a variety of client architectures including Rich Internet clients based on Flex, Silverlight and Javacript, occasionally connected Mobile Devices that are used for field work, as well as Desktop clients built using .Net and Java. The key to success is simple and elegant Web APIs and online SDKs that allow customers to easily exploit the value of the information managed by their servers. This talk will highlight specific areas of interest to researchers working on spatial and temporal databases as it spans the above aspects of GIS server technology. 